\title{
Severe Thrombocytosis and Anemia Associated With Celiac Disease in a Young Sudanese Male
}

\author{
Sulaf I Abdelaziz ${ }^{*}$, Khairi N K M² and Huda H Satti ${ }^{3}$ \\ ${ }^{1}$ Head department of Medicine, Soba University Hospital, Sudan \\ ${ }^{2}$ Consultant Internal Medicine and Endocrine, Soba University Hospital, Sudan \\ ${ }^{3}$ Assistant Professor, Faculty of Medicine, University of Khartoum, Sudan
}

Received: April 02, 2018; Accepted: April 06, 2018; Published: April 27, 2018

*Corresponding author: Sulaf I Abdelaziz, Head department of Medicine, Soba University Hospital, Sudan, E-mail: sulafibrahim1@gmail.com

\begin{abstract}
Thrombocytosis, A common hematologic finding, can present as an incidental finding and pose a great diagnostic challenge. Thrombocytosis can be due to reactive process or due to primary clonal disorder. Platelet count more than $1,000 \times 10^{3} / \mathrm{mm}^{3}$ is usually caused by clonal disorder. Reactive causes like iron deficiency anemia rarely lead to platelet counts more than $700 \times 10^{3} / \mathrm{mm}^{3}$. Here we report a case of a young man presenting with celiac disease associated with thrombocytosis with a platelet count of $1,489 \times 10^{3} / \mathrm{mm}^{3}$.
\end{abstract}

Keywords: Thrombocytosis; Celiac disease; Iron deficiency anemia;

\section{Introduction}

Thrombocytosis is commonly encountered in clinical settings with the majority of cases discovered incidentally. Thrombocytosis can be attributed to autonomous (neoplastic) overproduction or as a reactive overproduction secondary to infections, iron deficiency anemia or malignancies [1]. Iron deficiency anemia usually results in mild to moderate degree of reactive thrombocytosis [2]. The mechanism leading to secondary or reactive thrombocytosis is not completely understood. The accelerated megakaryopoiesis may result due to elevated megakaryocytic growth factors such as thrombopoiet in, Interleukin (IL)-6 or IL-11 [3]. But despite many efforts exact cause behind enhanced megakaryopoiesis is vaguely known and further investigation is required.

\section{Case report}

A 26 year old male presented with abdominal pain and yellow discoloration of his sclera for three weeks. He had history of splenectomy two years ago due to periportal fibrosis and portal hypertension and was transfused with one unit of packedred cells one week prior to admission as he was found to have anaemia.

On examination, patient was emaciated, pallor and icterus were present, no petechiae, ecchymosis or purpuric lesions were noted. Central abdominal tenderness was present, no organomegally and no ascites.
Work up of the abdominal pain revealed portal vein thrombosis and was started on anticoagulation.

His haemoglobin was $7 \mathrm{~g} / \mathrm{dl}$ (Normal range12-15g/dl), platelet count was $1,489 \times 10^{3} / \mathrm{mm}^{3}$ (normal range $150-450 \times 10^{3}$ / $\mathrm{mm}^{3}$ ).Total leucocyte count and differential leucocyte count were within normal range, mean corpuscular volume was $67.9 \mathrm{fl}$ (Normal range 80-100 fl), mean corpuscular haemoglobin was $18.4 p g$ (Normal range 27-32pg),mean corpuscular haemoglobin concentration was $27.2 \mathrm{~g} / \mathrm{dl}$ (Normal range 32-37g/dl). Iron studies revealed serum iron $3.3 \mu \mathrm{g} / \mathrm{dl}$ (Normal range $11-34.5 \mu \mathrm{g} /$ dl), serum ferritin was 41.8ng/dl (Normal range 30-400 ng/ dl) and Total Iron Binding Capacity 391 $\mathrm{g} / \mathrm{dl}$ (Normal range $125-345 \mu \mathrm{g} / \mathrm{dl}$ ). Results of iron studies were consistent with iron deficiency anemia. The peripheral blood smear showed microcytic hypochromic cells, target cells with increased number of platelets.

Bone marrow aspiration study revealed hyper active erythropoies is with micro normoblastic maturation and megakaryocytic hyperplasia. Trephine biopsy showed cellular marrow with megakaryocytes and no abnormal cells. Overall bone marrow picture doesn't support diagnosis of essential thrombocythemia. Megakaryotes were normal in morphology, giant megakaryocytes were absent and Megakaryocytes did not show clustering. JAK2 mutation was negative. Ultrasound showed acute portal vein thrombosis (PVT) and diffuse periportal fibrosis. Anti-tissue Transglutaminase ATTG (IgA) was highly positive for cealiac disease.

OGD was done which showed grade II oesphgealvarices. His coagulation parameters were normal apart from increase APTT due to therapeutic heparin.

Patient was transfused with one unit of packed red cells and was advised to take oral iron supplements and a gluten free diet. Three weeks after that his haemoglobin increased to $9.4 \mathrm{~g} / \mathrm{dl}$ and platelet count decreased to $600 \times 10^{3} / \mathrm{mm}^{3}$. 


\section{Discussion}

The clinical presentation of celiac disease is variable ranging from a "typical" form with diarrhea, steatorrhoea, weight loss and impaired development, to forms with much more subtle symptoms or even a complete lack of typical clinical symptoms $[3,6]$. The patient in this case did not suffer from any typical symptoms of celiac disease but was emaciated and found to have severe iron-deficiency anemia and developed thrombosis from the high platelets. In view of this an underlying hematologic disease was suspected initially. However, bone marrow smear, bone marrow biopsy and JAK2 mutation failed to provide support for the diagnosis of essential thombocytosis or other types of myeloproliferative disease. Furthermore, after transfusion of one pack of erythrocyte concentrate and iron supplements with a gluten free diet, the platelet count started to come down dramatically within three weeks.

According to the WHO criteria, this rules out the possibility of essential thombocytosis since a sustained elevation of platelets of at least $600 \times 103 / \mu$ is mandatory for this diagnosis [2]. As summarized by Halfdanarson et al [3], anemia and/or thrombocytosis may occur as clinical manifestations of celiac disease in some cases $[2,3]$.Thrombocytosis in celiac disease could be secondary to inflammation, iron deficiency or functional hypo-splenia [3]. In our case the patient had severe iron deficiency anemia probably caused by mal absorption or chronic blood loss from oesophageal varices. Interestingly, as stated by Sanchez and Ewton [2], in the case of iron deficiency as the underlying cause for thrombocytosis, the platelet count rarely exceeds $700 \times 10^{3} /$ $\mu \mathrm{l}[3]$. Thus, the case presented here is of iron-deficiency anemia associated with celiac disease with an unusually high platelet count. Although there appears to be an association between iron deficiency anemia and reactive thrombocytosis, the mechanism responsible is still a matter of debate [7-9]. Of interest in this respect is the notion that the amino acid sequence homology of thrombopoietin and erythropoietin might explain thrombocytosis in children with iron deficiency anemia [8]. Blood levels of erythropoietin are up regulated in response to anemia or arterial hypoxemia.

Juxtagloumerular interstitial cells of the renal cortex sense oxygen levels through oxygen-dependent prolyl hydroxylase. This controls the expression of hypoxia-inducible factor $1 \alpha$ (HIF-1 $\alpha$ ), the transcription factor for erythropoietin [10]. In women with iron deficiency, Akan et al [9] reported elevated erythropoietin levels associated with thrombocytosis which both normalized after iron substitution. In the seconds ub group of this study there was again a correlation between iron deficiency and erythropoietin levels; however, thrombocytosis in these patients was absent [9].An elevation of platelet count was observed in animal studies and in patients with renal failure receiving erythropoietinas medication [11]. Erythropoietin and thrombopoietin belong to the same hematopoietic growth factor subfamily. Taken together, it is tempting to speculate that elevated erythropoietin levels in patients with iron-deficiency anemia lead to thrombocytosis as a result of some kind of cross- reactivity at the level of the thrombopoietin receptor c-mpl because of the homology of some amino acid sequences of erythropoietin to thrombopoietin [8]. The clinical course and correlation of erythropoietin and platelet counts in our patient would be in agreement with this hypothesis. However, recent in vitro data from Broudy et al [12] provide evidence to the contrary as they found no cross-competition for binding of erythropoietin and thrombopoietin to c-mpl and the erythropoietin receptor. On the other hand, it has been suggested that erythropoietin and thrombopoietin can synergistically stimulate megakaryocyte proliferation owing to signaling of erythropoietin at the level of bipotenterythroid/megakaryocyte progenitor cells $[13,14]$.

Considering the data of Akan et al [9], the role of erythropoietin in thrombocytosis in patients with iron deficiency appears to be even more complex since not all patients with iron-deficiency anemia and elevated erythropoietin levels uniformly present with thrombocytosis. Thus, there has to be some kind of additional factors present in some iron-deficiency patients contributing to the stimulatory potential of erythropoietin on thrombopoiesis.

In conclusion, even in the presence of an 'atypical' high platelet count one should consider the possibility of reactive thrombocytosis. The exact mechanism of thrombocytosis in iron-deficiency anemia remains to be defined. Cross-reaction between erythropoietin and thrombopoietin receptors owing to structural homology is discussed by some groups but this is contradicted by recent molecular data showing no crosscompetition for binding of erythropoietin and thrombopoietin to c-mpl and the erythropoietin receptor. Recent data suggest a synergistic effect of erythropoietin and thrombopoietin on the level of bi-potenterythroid/megakaryocyte progenitor cells. However, this fails to explain why not all patients with irondeficiency anemia and elevated levels of erythropoietin present with thrombocytosis. Therefore, there must be additional, yet undefined mechanisms which contribute to the development of thrombocytosis in some patients with iron deficiency anemia.

\section{Conclusion}

This case establishes the association of an extreme thrombocytosis secondary to iron deficiency anemia. The fact to be stressed is that even in the presence of an extremely high platelet count possibility of reactive thrombocytosis should be considered and underlying cause should be investigated because in secondary thrombocytosis treatment of the underlying cause normalizes the platelet count.

\section{Authorship}

All authors have no conflict of interest to declare

\section{Acknowledgements}

SI Abdelaziz, idea, work up of the patient, edited and revised the paper

N K M Khairi, wrote the paper

H H Satti, revised the paper 


\section{References}

1. Buss DH, CashellAW, O'Connor ML, Richards F II, Case LD. Occurrence etiology, and clinical significance of extreme thrombocytosis: a study of 280 cases. Am J Med. 1994;96(3):247-253.

2. Dan K. Thrombocytosis in iron deficiency anemia. Intern Med. 2005;44:1025-1026.

3. Ceresa IF, Noris P, Ambaglio C, Pecci A, Balduini CL. Thrombopoietin is not uniquely responsible form thrombocytosis in inflammatory disorders. Platelets. 2007;18(8):579-582.

4. Buss DH, Cashell AW, O'Connor ML, Richards F. Occurrence, etiology and clinical significance of extreme thrombocytosis: a

study of 280 cases. Am J Med. 1994;96:247-253.

5. Sanchez S, Ewton A. Essential thrombocythemia: a review of diagnostic and pathologic features. Arch Pathol Lab Med. 2006 Aug;130(8):1144-1150.

6. Halfdanarson TR, Litzow MR, Murray JA. Hematologic manifestations of celiac disease. Blood. 2007;109(2):412-421.

7. Carroccio A, Giannitrapani L, Di Prima L, Iannitto E, Montalto G, Notarbartolo A. Extreme thrombocytosis as a sign of celiac disease in the elderly: case report. Eur J GastroenterolHepatol. 2002;14(8):897-900.

8. Fasano A. Celiac Disease - How to Handle a Clinical Chameleon. N Engl J Med. 2003;348(25):2568-2570.

9. Bottaro G, Cataldo F, Rotolo N, Spina M, Corazza GR. The clinical pattern of subclinical/silent celiac disease: an analysis on 1026 consecutive cases. Am J Gastroenterol. 1999;94(3):691- 696.
10. Schafer AI. Thrombocy tosis. N Engl J Med. 2004;350(12):1211-1219.

11. Bilic E, Bilic E. Amino acid sequence homology of thrombopoietin and erythropoietin may explain thrombocytosis in children with iron deficiency anemia. J Pediatr Hematol Oncol. 2003;25(8):675676.

12. Akan H, Guven N, Aydogdu I, Arat M, Beksac M, Dalva K. Thrombopoietic cytokines in patients with iron deficiency anemia with or without thrombocytosis. Acta Haematol. 2000;103(3):152156.

13. Kaushansky K. Lineage-specific hematopoietic growth factors. N Engl J Med. 2006;354(19):2034-2045.

14. Loo M, Beguin Y. The effect of recombinant human erythropoietin on platelet counts is strongly modulated by the adequacy of iron supply. Blood. 1999;93(10):3286-3293.

15. Broudy VC, Lin N, Fox N. Hematopoietic cells display high affinity receptors for thrombopoietin. 1997;89(6):1896-1904.

16.Cardier JE, Erickson-Miller CL, Murphy MJ Jr. Differential effect of erythropoietin and GM-CSF on megakaryocytopoiesis from primitive bone marrow cells in serum-free conditions. Stem Cells. 1997;15(4):286-290.

17. Broudy VC, Lin NL, Kaushansky K. Thrombopoietin (c-mplLIgand) Acts Synergistically With Erythropoietin, Stem Cell Factor, and Interleukin-11 to Enhance Murine Megakaryocyte Colony Growth and Increases Megakaryocyte Ploidy In Vitro. Blood. 1995;85(7):1719-1726. 